\title{
Finding a majority ball with majority answers
}

\author{
Dániel Gerbner* Balázs Keszegh ${ }^{\dagger} \quad$ Dömötör Pálvölgyi ${ }^{\ddagger}$

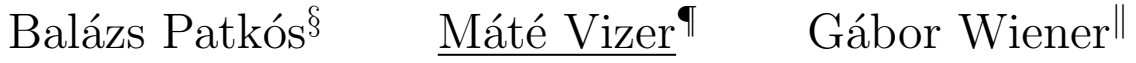

May 25, 2015

\begin{abstract}
Suppose we are given a set of $n$ balls $\left\{b_{1}, \ldots, b_{n}\right\}$ each colored either red or blue in some way unknown to us. To find out some information about the colors, we can query any triple of balls $\left\{b_{i_{1}}, b_{i_{2}}, b_{i_{3}}\right\}$. As an answer to such a query we obtain (the index of) a majority ball, that is, a ball whose color is the same as the color of another ball from the triple. Our goal is to find a non-minority ball, that is, a ball whose color occurs at least $\frac{n}{2}$ times among the $n$ balls. We show that the minimum number of queries needed to solve this problem is $\Theta(n)$ in the adaptive case and $\Theta\left(n^{3}\right)$ in the non-adaptive case.
\end{abstract}

\section{Introduction}

This paper deals with search problems where the input is a set of $n$ balls, each colored in some way unknown to us and we have to find a ball possesing a certain property (or show that such a ball does not exist) by asking certain queries. Our goal is to determine the minimum number of queries needed in the worst case. It is possible that the queries are all fixed beforehand (in which case we speak of non-adaptive search) or each query might

*MTA Alfréd Rényi Institute of Mathematics, Reáltanoda u. 13-15. Budapest, 1053 Hungary. Email: gerbner.daniel@mta.renyi.hu. Research supported by Hungarian Scientific Research Fund (OTKA), under grant PD 109537.

${ }^{\dagger}$ MTA Alfréd Rényi Institute of Mathematics, Reáltanoda u. 13-15. Budapest, 1053 Hungary. Email: keszegh.balazs@mta.renyi.hu. Research supported by Hungarian Scientific Research Fund (OTKA), under grant PD 108406, and by the János Bolyai Research Scholarship of the Hungarian Academy of Sciences.

${ }_{\ddagger}^{\ddagger}$ Eötvös Loránd University, Department of Computer Science. Pázmány Péter sétány 1/C Budapest, 1117 Hungary. Email:dom@cs.elte.hu. Research supported by Hungarian Scientific Research Fund (OTKA), under grant PD 104386, and by the János Bolyai Research Scholarship of the Hungarian Academy of Sciences.

§MTA-ELTE Geometric and Algebraic Combinatorics Research Group. Pázmány Péter sétány 1/C Budapest, 1117 Hungary. Email:patkosb@cs.elte.hu. Research supported by the János Bolyai Research Scholarship of the Hungarian Academy of Sciences.

『MTA Alfréd Rényi Institute of Mathematics, Reáltanoda u. 13-15 Budapest, 1053 Hungary. Email: vizermate@gmail.com

"Budapest University of Technology and Economics. Email:wiener@cs.bme.hu. Research was supported by grant no. OTKA 108947 of the Hungarian Scientific Research Fund and by the János Bolyai Research Scholarship of the Hungarian Academy of Sciences. 
depend on the answers to the earlier queries (in which case we speak of adaptive search). We say that a ball $b$ is a majority ball of a set $A$ if there are more than $\frac{|A|}{2}$ balls in the set that have the same color as $b$. Similarly, a ball $b$ is a non-minority ball of a set $A$ if there are at least $\frac{|A|}{2}$ balls in the set that have the same color as $b$. Note that these two notions are different if and only if $n$ is even and $\frac{|A|}{2}$ balls are colored of the same color, in which case each of these balls is a non-minority ball and there are no majority balls (moreover, if there are only two colors, then the size of the other color class is also $\frac{|A|}{2}$, thus every ball is a non-minority ball). For more than two colors it is possible that even non-minority balls do not exist. A ball $b$ is said to be a plurality ball of a set $A$ if the number of balls in the set with the same color as $b$ is greater than the number of balls with any of the other colors. In this paper we focus on the case of just two colors.

The most natural non-trivial question is the so-called majority problem which has attracted the attention of many researchers. In this problem our goal is to find a majority ball (or show that none exists), such that the possible queries are pairs of balls $\left\{b_{1}, b_{2}\right\}$ and the answer tells us whether $b_{1}$ and $b_{2}$ have the same color or not. In the adaptive model, Fisher and Salzberg [6] proved that $\lfloor 3 n / 2\rfloor-2$ queries are necessary and sufficient for any number of colors, while Saks and Werman [11] showed that if the number of colors is known to be two, then the minimum number of queries is $n-b(n)$, where $b(n)$ is the number of 1's in the binary representation of $n$ (simplified proofs of the latter result were later found, see $[2,9,12])$. In the non-adaptive model with two colors, it is easy to see that the minimum number of queries needed is $n-1$ if $n$ is even and $n-2$ if $n$ is odd.

There are several variants of the majority problem [1]. The plurality problem, where we have to find a plurality ball (or show that none exists) was considered, among others, in $[1,5,7]$. Another possible direction is to use sets of size greater than two as queries [4], [3].

\section{Models, results}

Now we define the main model studied in this paper. In the original comparison model the answer to the query $\left\{b_{1}, b_{2}\right\}$ can be interpreted as the answer to the question whether there is a majority ball in the subset $\left\{b_{1}, b_{2}\right\}$. If the answer is yes, then obviously both $b_{1}$ and $b_{2}$ are majority balls. Therefore we obtain a generalization of the comparison model if for any query that is a subset of the balls the answer is either (the index of) a majority ball, or that there is no majority ball in the given subset (which cannot be the case if the size of the subset is odd and there are only two colors). We study this model in case of two colors, and mostly when only queries of size three are allowed, although we also prove some results for greater query sizes.

Unfortunately, even asking all triples cannot guarantee that we can solve the majority problem for two colors. Suppose we have an even number of balls that are partitioned into two sets of the same size, $X$ and $Y$, and suppose that the answer for any triple $T$ is a ball from $T \cap X$ if and only if $|T \cap X| \geq 2$. In this case we cannot decide whether all balls have the same color or all balls in $X$ are red, but all balls in $Y$ are blue. In the former case all balls are majority balls, while in the latter there exists no majority ball.

Because of this, our aim will be to show a non-minority ball (which always exists if there 
are only two colors). Let us assume the balls are all red or blue and all queries are of size $q$. We will denote the minimum number of queries needed (in the worst case) to determine a non-minority ball by $A_{q}(n)$ in the adaptive model and by $N_{q}(n)$ in the non-adaptive model.

At first sight the model we have just introduced seems to be rather artificial. Let us, however, state a more natural problem that is equivalent to this model.

Suppose that our input is a binary sequence of length $n$, i.e., $n$ numbers such that each is either 0 or 1 . Our task is to find a median element, such that the queries are odd subsets of the input elements and the answer is one of the the median elements of the subset. Let us assume queries are of size $q$. Denote the the minimum number of queries needed in the worst case to determine a median element by $A_{q}^{\text {med }}(n)$ in the adaptive model and by $N_{q}^{\text {med }}(n)$ in the non-adaptive model. If we replace 0 and 1 by red and blue, one can easily see that the median elements of any set are exactly the non-minority balls of the set. So we have $A_{2 l+1}(n)=A_{2 l+1}^{\text {med }}(n)$ and $N_{2 l+1}(n)=N_{2 l+1}^{\text {med }}(n)$.

We obtain a natural generalization that also works for even sized subsets if the answer is the $t$ th element for some fixed $t$. More precisely, for a query $Q$, the answer may be $a$ if and only if there exists $t-1$ elements $e \in Q \backslash\{a\}$, such that $e \geq a$ and $|Q|-t$ elements $e \in Q \backslash\{a\}$, such that $q \leq a$. Note that in this model there might be more than one valid answers to a given query. This can be outruled by assuming that all elements are different (in which case we do not deal with just the numbers 0 and 1, obviously). This approach was proposed by G. O.H. Katona and studied by Johnson and Mészáros [10]. They have shown that if all elements are different, then they can be almost completely sorted ${ }^{1}$ using $O(n \log n)$ queries in the adaptive model and $O\left(n^{q-t+1}\right)$ queries in the non-adaptive model and both results are sharp. However, their algorithms fail if not all elements are different. Our results imply that the same bound holds in the adaptive model with no restriction. However, the bound in the non-adaptive model cannot be extended to the general case and we prove some related results.

As we have already mentioned, we will mainly consider the case $k=3$ with two colors. To state our results concerning $A_{3}(n)$ and $N_{3}(n)$ we introduce the following notations. We write $[n]=\{1,2, \ldots, n\}$ for the set of the first $n$ positive integers and the set of balls is denoted by $B=[n]$. For a set $S$, the set of its $k$-subsets will be $\operatorname{denoted}$ by $\left(\begin{array}{l}S \\ k\end{array}\right)$. Let $\mathcal{Q} \subseteq\left(\begin{array}{l}B \\ 3\end{array}\right)$ be a query set. Then for any ball $b \in B$ let $d_{\mathcal{Q}}(b)=|\{Q \mid b \in Q \in \mathcal{Q}\}|$ denote the degree of $b$ in $\mathcal{Q}$ and for any two balls $b_{i}, b_{j} \in B$ let $d_{\mathcal{Q}}\left(b_{i}, b_{j}\right)=\left|\left\{Q \mid\left\{b_{i}, b_{j}\right\} \subset Q \in \mathcal{Q}\right\}\right|$ denote the co-degree of $b_{i}$ and $b_{j}$ in $\mathcal{Q}$. Furthermore, let us write $\delta(\mathcal{Q})=\min \left\{d_{\mathcal{Q}}(b) \mid b \in B\right\}$ and $\delta_{2}(\mathcal{Q})=\min \left\{d_{\mathcal{Q}}\left(b_{i}, b_{j}\right) \mid b_{i}, b_{j} \in B\right\}$.

Theorem 2.1. $A_{3}(n)=O(n)$.

Theorem 2.2. (i) If $n$ is odd, then $\frac{1}{2}\left(\begin{array}{l}n \\ 3\end{array}\right) \leq N_{3}(n)$. Moreover, if $\mathcal{Q} \subseteq\left(\begin{array}{l}B \\ 3\end{array}\right)$ is a set of queries such that $\delta_{2}(\mathcal{Q})<\frac{n-2}{2}$, i.e., there is a pair of balls $x, y$ with $d_{\mathcal{Q}}(x, y)<\frac{n-2}{2}$, then $\mathcal{Q}$ cannot determine a non-minority ball.

(ii) There exists a non-adaptive query set $\mathcal{Q} \subseteq\left(\begin{array}{l}B \\ 3\end{array}\right)$ with $\delta_{2}(\mathcal{Q})=\lfloor n / 2\rfloor+1$ that determines a non-minority ball.

\footnotetext{
${ }^{1}$ Note that the $t-1$ largest and the $q-t+1$ smallest elements cannot ever be differentiated with such questions, so we only want to determine these and sort the rest.
} 
(iii) If $n$ is even, then $\frac{1}{8}\left(\begin{array}{l}n \\ 3\end{array}\right) \leq N_{3}(n)$. Moreover, if $\mathcal{Q} \subseteq\left(\begin{array}{l}B \\ 3\end{array}\right)$ is a set of non-adaptive queries such that there exist four balls $x, y, u, v$ with $d_{\mathcal{Q}}(x, u)+d_{\mathcal{Q}}(x, v)+d_{\mathcal{Q}}(y, u)+d_{\mathcal{Q}}(y, v) \leq$ $n / 2-3$, then $\mathcal{Q}$ cannot determine a non-minority ball.

Parts (i) and (ii) of Theorem 2.2 shows that the minimum value of $\delta_{2}(\mathcal{Q})$ taken over all non-adaptive sets of queries determining a non-minority ball differs from $n / 2$ by at most 1 provided $n$ is odd. We conjecture that this holds independently of the parity of $n$ (maybe with a larger constant in the role of 1 ).

Theorem 2.3. (i) $N_{3}(n) \leq\left(\frac{5}{6}+o(1)\right)\left(\begin{array}{l}n \\ 3\end{array}\right)$. Moreoever, any non-adaptive query set $\mathcal{Q}$ with $\delta_{2}(\mathcal{Q})>\frac{5 n}{6}$ determines a non-minority ball.

(ii) There exists a non-adaptive query set $\mathcal{Q}$ with $\delta_{2}(\mathcal{Q})=\frac{5 n}{6}-3$ that does not determine a non-minority ball.

We have some information on the non-minority problem if the query size $q>3$ (but the number of colors is still only two) and also investigate some related models. Recall that in this model a query is $q$ balls, and the answer is either (the index of) a majority ball, or that there is no majority color in this subset (if the size of the subset is odd and there are only two colors, then this cannot happen), and that we denote the number of queries needed to determine a non-minority ball by $A_{q}(n)$ in the adaptive model and by $N_{q}(n)$ in the non-adaptive model. As many of the proofs and algorithms are very similar to the ones we used for the case $q=3$, we do not always go into all the details.

By definition, the model is quite different for odd and even $q$. We first consider the case when $q$ is odd, where we could only prove the following results.

Theorem 2.4. $N_{2 l+1}(n)=\Omega\left(n^{3}\right), A_{2 l+1}(n)=O\left(n^{2}\right)$.

Now we consider the case $q=2 l$. We have the following results.

Theorem 2.5. $N_{2 l}(n) \leq n(n-2 l), A_{2 l}(n) \leq n-2 l+1$.

The huge difference between the odd and even cases motivates us to consider the model where the answer to a query is simply a non-minority ball. We define the following more general model. For some $0<\alpha \leq 1 / 2$, we say that a ball $b \in B$ is an $\alpha$-ball of the set $B$ if there are at least $\alpha(|B|-1)$ other balls in the set that have the same color as $b$. Similar density queries were considered in the group testing model in [8]. Notice that a $\frac{1}{2}$-ball is just a majority ball. In this new model, the queries are subsets of size $q$ of the set of $n$ balls colored with two colors and the answer we obtain to a query $Q$ is an $\alpha$-ball of $Q$ or that there is no such ball in the set.

Our goal is to find a ball having the same color as many other balls. Let $A$ be the largest integer, such that $\left\lceil\frac{q}{A}\right\rceil-1 \geq \alpha(q-1)$.

Theorem 2.6. For any $q, \alpha$ and $n$, if $A$ divides $n$ or $q$, or $(n \bmod A)<(q \bmod A)$, then even asking all the possible $\left(\begin{array}{l}n \\ q\end{array}\right)$ queries, it is possible that we cannot show a $\frac{1}{A}$-ball of the full set.

The following theorem shows that we can find an $\alpha$-ball for every other instances of $q$ and $\alpha$ if $n$ is large enough. Moreover, $\frac{1}{A}$ cannot be replaced by any smaller number.

Theorem 2.7. After asking all the possible $\left(\begin{array}{l}n \\ q\end{array}\right)$ queries, we can show an almost $\frac{1}{A}$-ball of the full set, a ball such that there are at least $\frac{n}{A}-c_{q}$ other balls of the same color, where $c_{q}$ is a constant depending only on $q$. 


\section{References}

[1] M. Aigner. Variants of the majority problem. Discrete Applied Mathematics, 137(1):325, 2004.

[2] L. Alonso, E. M. Reingold, and R. Schott. Determining the majority. Information Processing Letters, 47(5):253-255, 1993.

[3] G. De Marco and E. Kranakis. Searching for majority with k-tuple queries. Discrete Mathematics, Algorithms and Applications, to appear.

[4] G. De Marco, E. Kranakis, and G. Wiener. Computing majority with triple queries. Theor. Comput. Sci., 461:17-26, 2012.

[5] Z. Dvorak, V. Jelínek, D. Král, J. Kyncl, and M. E. Saks. Probabilistic strategies for the partition and plurality problems. Random Struct. Algorithms, 30(1-2):63-77, 2007.

[6] M. J. Fischer and S. L. Salzberg. Finding a majority among $n$ votes. Journal of Algorithms, 3:375-379, 1982.

[7] D. Gerbner, G. O. Katona, D. Pálvölgyi, and B. Patkós. Majority and plurality problems. Discrete Applied Mathematics, 161(6):813-818, 2013.

[8] D. Gerbner, B. Keszegh, D. Pálvölgyi, and G. Wiener. Density-based group testing. In Information Theory, Combinatorics, and Search Theory - In Memory of Rudolf Ahlswede, pages 543-556, 2013.

[9] T. P. Hayes, S. Kutin, and D. van Melkebeek. The quantum black-box complexity of majority. Algorithmica, 34(4):480-501, 2002.

[10] R. Johnson and G. Mészáros. Sorting using non-binary comparisons. Unpublished manuscript.

[11] M. E. Saks and M. Werman. On computing majority by comparisons. Combinatorica, 11(4):383-387, 1991.

[12] G. Wiener. Search for a majority element. Journal of statistical planning and inference, 100(2):313-318, 2002. 